\title{
An Improved Algorithm for Pseduo-Jacobi-Fourier Moments
}

\author{
Guleng Amu \\ Department of Physics, Inner Mongolia Agricultural University, Huhhot, China \\ Email: amoolg@163.com
}

How to cite this paper: Amu, G. (2017) An Improved Algorithm for Pseduo-JacobiFourier Moments. Optics and Photonics Journal, 7, 68-74.

https://doi.org/10.4236/opj.2017.78B009

Received: May 27, 2017

Accepted: August 7, 2017

Published: August 10, 2017

\begin{abstract}
Image moments have been used in many research fields of the engineering. However, the related computation of invariant moments mostly adopted the polar coordinate system, which not only increase the computational load, but also cause large quantized error. To solve this problem, an improved algorithm to compute Pseudo-Jacobi-Fourier moments in the Cartesian coordinate system is proposed in this paper. The experimental results show that the reconstructed image with improved PJFM's has more advantages than polar coordinate system, such as more information, fewer moments, less time consuming. And the recognition rate of the microscopic images of 8 helminth eggs was also higher than in polar coordinate system.
\end{abstract}

\section{Keywords}

Invariant Moments, Cartesian Coordinate System, Image Reconstruction

\section{Introduction}

Image moments, because of their powerful description of the image content, have been used in many research fields of the engineering, such as image processing [1] [2], pattern recognition and machine vision [3]. The first introduction of image moments for classification purposes was performed by $\mathrm{Hu}$ [4], and then developed into other families, such as Legendre [5], Zernike [6], PseudoZernike [7], Fourier-Mellin [8], Tchebichef [9], Krawtchouk [10], Pseudo-Jacobi-Fourier[11] moment. These moments can be used as image descriptors after an appropriate normalization procedure in order to achieve translation, scale and rotation invariance. However, the related computation for these moments mostly adopted the polar coordinate system, which not only increase the computational load, but also cause large quantized error [12] [13]. To solve this 
problem, an improved algorithm to compute Pseudo-Jacobi-Fourier moments in the Cartesian coordinate system is proposed in this paper. This improved algorithm is applied to classify the microscopic images of helminth eggs by using Euclidean distance classifier [14], and the recognition rate is $92.2 \%$.

\section{Improved Algorithm for Pseduo-Jacobi-Fourier Moments}

\subsection{Definition of Pseduo-Jacobi-Fourier Moments}

Bhatia and Wolf have shown [15] that a polynomial that is invariant in form for any rotation of axes about the origin must be of the form

$$
V(r \cos \theta, r \sin \theta)=R_{n}(r) \exp (j m \theta)
$$

where $R_{n}(r)$ is a radial polynomial in $r$ of degree $n$. We now defined a new set of orthogonal moments, Pseudo-Jacobi $(p=4, q=3)$-Fourier Moments (PJFM's), based on Jacobi polynomials. In the polar coordinates $(r, \theta)$, radial Jacobi polynomials $G_{n}(p, q, r)$ are expressed as

$$
\begin{aligned}
G_{n}(p, q, r)=\frac{\Gamma(q+n)}{\Gamma(p+2 n)} \sum_{m^{\prime}=0}^{n}(-1)^{m} \times\left(\begin{array}{l}
n \\
m^{\prime}
\end{array}\right) \frac{\Gamma\left(p+2 n-m^{\prime}\right)}{\Gamma\left(q+n-m^{\prime}\right)} r^{n-m^{\prime}} \\
(p-q>-1, q>0)
\end{aligned}
$$

where the function $G_{n}(p, q, r)$ is orthogonal over the range [0,1]

$$
\int_{0}^{1} G_{n}(r) G_{k}(r) w(r) d r=b_{n} \delta_{n k}
$$

where $\delta_{n k}$ is Kronecker symbol, and

$$
\begin{aligned}
w(r)=(1-r)^{p-q} r^{q-1} & \\
b_{n}(p, q) & =\frac{n ! \Gamma(n+q) \Gamma(n+p)}{(2 n+p)} \times \frac{\Gamma(n+p-q+1)}{\Gamma^{2}(2 n+p)}
\end{aligned}
$$

The radial polynomials of OFMM's, CHM's and ZM's belong to Jacobi polynomials with $p=q=2, p=q=1 / 2$ and $p=q=m+1$ respectively. When $p=4, q=3$, radial Jacobi polynomials become $G_{n}(4,3,0)=0$, so Pseudo-Jacobi polynomials $J_{n}(r)$ are defined as

$$
J_{n}(r)=(-1)^{n} \sqrt{\frac{(2 n+4)}{(n+3)(n+1)}(1-r)} r \times \sum_{s=0}^{n}(-1)^{s} \frac{(n+s+3) !}{(n-s) ! s !(s+2) !} r^{s}
$$

$\int_{0}^{1} J_{n}(r) J_{k}(r) r d r=\delta_{n k}$

So a new set of orthogonal polynomial function $P_{n m}(r, \theta)$, which consists of radial function $J_{n}(r)$ and angular function $\exp (j m \theta)$, is obtained as

$$
P_{n m}(r, \theta)=J_{n}(r) \exp (j m \theta)
$$

obviously, the $P_{n m}(r, \theta)$ is orthogonal over the range $[0,1]$.

$$
\int_{0}^{2 \pi} \int_{0}^{1} P_{n m}(r, \theta) P_{k l}(r, \theta) r d r d \theta=\delta_{n m k l}
$$

According to the orthogonal theory, the image function $f(r, \theta)$ can be written 
as an infinite series expansion in terms of $P_{n m}(r, \theta)$

$$
f(r, \theta)=\sum_{n=0}^{\infty} \sum_{m=-\infty}^{+\infty} \phi_{n m} J_{n}(r) \exp (j m \theta)
$$

where

$$
\phi_{n m}=\frac{1}{2 \pi} \int_{0}^{2 \pi} \int_{0}^{1} f(r, \theta) J_{n}(r) \exp (-j m \theta) r d r d \theta
$$

then $\phi_{n m}$ is defined as Pseudo-Jacobi-Fourier Moments (PJFM's), and $r=1$ is the maximum size of the objects that can be encountered in a particular application.

Actually, most of the images are defined in the Cartesian coordinate system. When calculating PJFM's, the images need to be converted to polar coordinates, so may cause some problems: 1) Quantization error is introduced into the calculation of PJFM's, which increases calculating amount and additional noise; 2) In polar coordinates, the discrete points near from the origin are more than those in the Cartesian coordinate system, which lead to information redundancy; 3) Far from the origin, the scattered points in the polar coordinate system are less than those in the Cartesian coordinate system, which lead to information loss. Therefore, an improved algorithm for computing PJFM's Cartesian coordinate system is developed in this paper.

\subsection{Improved Algorithm for Pseduo-Jacobi-Fourier Moments}

When calculating PJFM's in the Cartesian coordinates, the image should first be normalized into the unit circle, and the integral region of Equation (11) as shown in Figure 1.

Substitute Equation (6) into Equation (8)

$$
P_{n m}(r, \theta)=J_{n}(r) e^{-j m \theta}=a_{n} \sqrt{(1-r) r} \sum_{s=0}^{n} b_{n s} r^{s} e^{-j m \theta}
$$

where

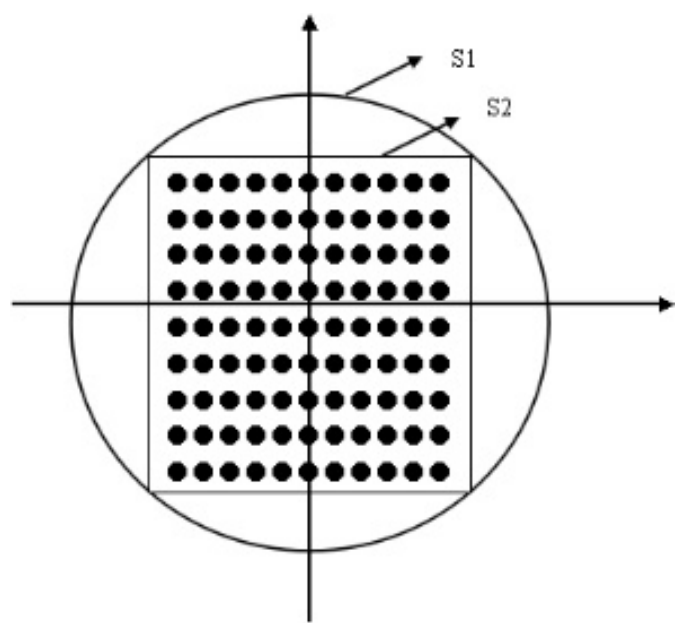

Figure 1. The integral region of PJFM. 


$$
r^{s} e^{-j m \theta}=(x+i y)^{\alpha}(x-i y)^{\beta} \text { and } \alpha=\frac{s-m}{2}, \beta=\frac{s+m}{2}
$$

Substitute Equation (13) and $r=\sqrt{x^{2}+y^{2}}$ into Equation (12)

$$
\begin{aligned}
& J_{n}(r) e^{-j m \theta}=\sum_{s=0}^{n} a_{n} b_{n s} \sqrt{\left(x^{2}+y^{2}\right)^{1 / 2}-\left(x^{2}+y^{2}\right)}(x+i y)^{\alpha}(x-i y)^{\beta} \\
& \varphi_{n m}=\frac{1}{2 \pi} \int_{0}^{2 \pi} \int_{0}^{1} f(r, \theta) P_{n m}(r, \theta) r d r d \theta=\frac{1}{2 \pi} \iint_{s 1} f(r, \theta) P_{n m}(r, \theta) d s \\
& =\frac{1}{2 \pi} \iint_{s 2} f(r, \theta) P_{n m}(r, \theta) d s+\frac{1}{2 \pi} \iint_{s 1-s 2} f(r, \theta) P_{n m}(r, \theta) d s
\end{aligned}
$$

Figure 1 shows that $s 1-s 2$ is not the part of the image and there is no pixel over it, Equation (15) can be written as

$$
\begin{aligned}
\varphi_{n m} & =\frac{1}{2 \pi} \iint_{s 2} f(x, y) J_{n}(r) e^{-j m \theta} d x d y \\
& =\frac{1}{2 \pi} \iint_{s 2} f(x, y) J_{n}\left(\sqrt{x^{2}+y^{2}}\right) e^{-j m\left[\arctan \left(\frac{y}{x}\right)+k \pi\right]} d x d y
\end{aligned}
$$

So Equation (16) is the general formula for calculating PJFM's in Cartesian coordinate system.

\subsection{Image Reconstruction Using Improved Algorithm}

Image reconstruction can be used as an effective means to evaluate the quality of feature extraction. The more PJFM's used to reconstruct images, the closer to the original image. An image of capital E, shown in Figure 2, is reconstructed by Equation (17).

$$
\hat{f}(r, \theta)=\sum_{n=0}^{N} \sum_{m=-M}^{M} \Phi_{n m} J_{n}(r) e^{j m \theta}
$$

The four corners of the reconstructed image are black and some pixels have been lost in the Figure 3. But the black area of the four corners of the reconstructed image is shrinking with the increase of $\mathrm{N}$ and $\mathrm{M}$, and the edge information is well preserved in the Figure 4. Figure 4 also shows that $\mathrm{E}$ is differentiated well when $\mathrm{N}=\mathrm{M}=6$ instead of $\mathrm{N}=\mathrm{M}=10$ as in Figure 3.

As for computation time, reconstruction time in the Cartesian coordinate system is much shorter than the polar coordinates system, shown in Table 1.

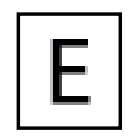

Figure 2. The original image of $\mathrm{E}$.

Table 1. Reconstruction time comparison.

\begin{tabular}{cccccc}
\hline $\mathrm{N}=\mathrm{M}$ & 1 & 5 & 10 & 15 & 20 \\
\hline Cartesian (s) & 0.5 & 1.3 & 3.8 & 5.6 & 7.4 \\
Polar (s) & 2 & 36 & 76 & 134 & 360 \\
\hline
\end{tabular}




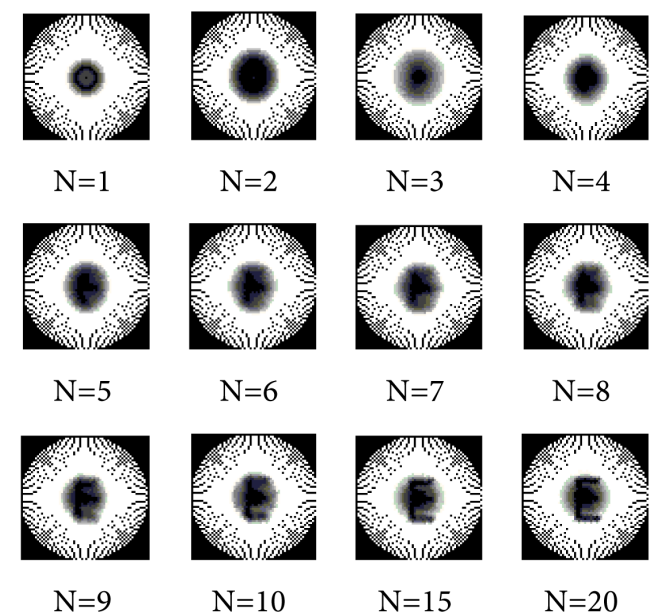

Figure 3. Reconstructed images in polar coordinates.

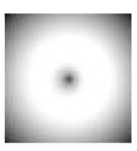

$$
\mathrm{N}=1
$$

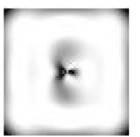

$\mathrm{N}=5$

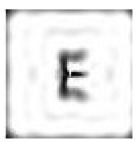

$\mathrm{N}=9$

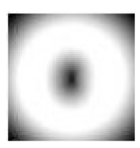

$\mathrm{N}=2$

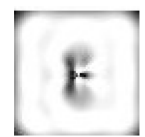

$\mathrm{N}=6$

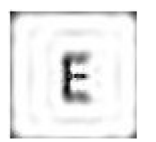

$\mathrm{N}=10$

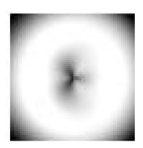

$\mathrm{N}=3$

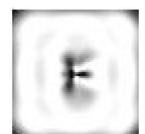

$\mathrm{N}=7$

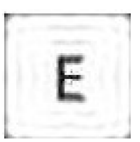

$\mathrm{N}=10$

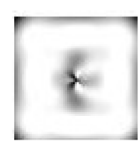

$\mathrm{N}=4$

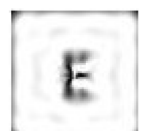

$\mathrm{N}=8$

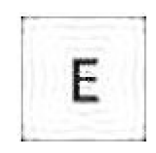

$\mathrm{N}=20$

Figure 4. Reconstructed images in Cartesian coordinates.

\subsection{Image Recognition Using Improved Algorithm}

In order to test the feature extraction performance of the improved PJFM's, a recognition experiment was done by using microscopic image of 8 kinds of helminth eggs, such as Fasciola hepatica (a), moniezia (b), Hairy ail nematode (c), paramphistomum (d), Nematodirus (e), Dicrocoelium chinensis (f), coccidium (g), Pancreatic Eurytrema (h).

The training sets consist of 20 different versions from each kind of helminth eggs, including 160 images. Testing sets consist of 307 untrained images from different version of 8 kinds of helminth eggs. Figure 5 gives a multi-distorted image of the microscopic image of some helminth eggs in a testing set.

Choosing $\mathrm{N}=\mathrm{M}=8$, the image feature extracted respectively by using Equation (16) in Cartesian coordinate system and Equation (11) in polar coordinate system, then the target objects were recognized by the minimum average distance rules . Euclidean distances are calculated by Equation (18), and the result is shown in Table 2. 

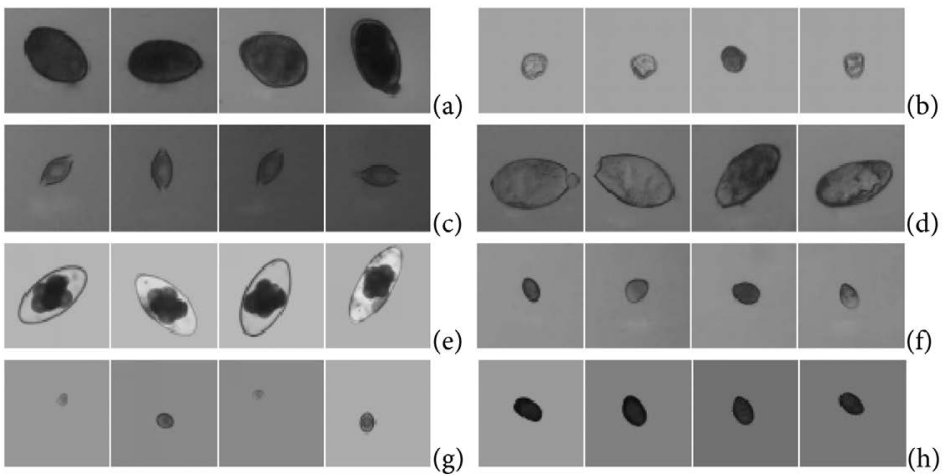

Figure 5. Part of the image of the experimental samples: (a) Fasciola hepatica; (b) moniezia; (c) Hairy ail nematode; (d) paramphistomum; (e) Nematodirus; (f) Dicrocoelium chinensis; (g) coccidium; (h) Pancreatic Eurytrema.

Table 2. Recognition result of parasite egg microscopic images

\begin{tabular}{cccccccccc}
\hline Types & (a) & (b) & (c) & (d) & (e) & (f) & (g) & (h) & \\
\hline Sample & 18 & 18 & 54 & 49 & 44 & 39 & 20 & 65 & 307 \\
& 18 & 17 & 51 & 42 & 44 & 30 & 18 & 63 & 283 \\
Cartesian (\%) & 100 & 94.4 & 94.4 & 85.7 & 100 & 76.9 & 90.0 & 96.9 & 92.2 \\
& 16 & 16 & 47 & 39 & 40 & 28 & 17 & 58 & 261 \\
Polar (\%) & 88.9 & 88.9 & 87.0 & 79.6 & 90.9 & 71.8 & 85.0 & 89.2 & 85.0 \\
\hline
\end{tabular}

$$
d_{i}=\left\{\sum_{\substack{n, m=0 \\ w h e n \\ m=0, n \neq 0,1}}^{10}\left[\left|\phi_{n m}\right|-\left(\left|\phi_{n m}\right|\right)_{i}\right]^{2}\right\}^{1 / 2}
$$

where $\left|\phi_{n m}\right|$ is the PJFM of the testing object, and $\left(\left|\phi_{n m}\right|\right)_{i}$ is the PJFM of the reference object of class $i$.

As can be seen from Table 2, the total recognition rate of the microscopic images of 8 helminth eggs was $92.2 \%$ with improved PJFM's, $7.2 \%$ higher than PJFM's in polar coordinate system.

\section{Conclusion}

An improved algorithm to compute Pseudo-Jacobi-Fourier moments in the Cartesian coordinate system is proposed in this paper. The experimental results show that the reconstructed image with improved PJFM's has more advantages than polar coordinate system, such as more information, fewer moments, less time consuming. And the recognition rate of the microscopic images of 8 helminth eggs was also higher than in polar coordinate system.

\section{Acknowledgements}

This work was supported by the National Natural Sciences Foundation of China [grant numbers 60967001 and 31060337]. 


\section{References}

[1] Lafta, H.L. and Abbas S.S. (2013) Effectiveness of Extended Invariant Moments in Fingerprint Analysis. Asian Journal of Computer and Information System, 1, 78-89.

[2] Mohammand, A. and Qusay, A.M. (2017) Efficient Image Recognition Technique Using Invariant Moments and Principle Component Analysis. Journal of Data Analysis and Information Processing, 5, 1-10. https://doi.org/10.4236/jdaip.2017.51001

[3] Papakostas, G.A., Karras, D.A., Mertzios, B.G. and Boutalis, Y.S. (2005) An Efficient Feature Extraction Methodology for Computer Vision Applications Using Wavelet Compressed Zernike Moments. International Journal on Graphics, Vision and Image Processing, Wavelets and Their Applications, SI1, 5-15.

[4] Hu, M.K. (1962) Visual Pattern Recognition by Moment Invariants. IRE Transactions on Information Theory, IT-8, 179-187.

[5] Mukundan, R. and Ramakrishnan, K.R. (1998) Moment Functions in Image Analysis. World Scientific Publisher, Singapore. https://doi.org/10.1142/3838

[6] Papakostas, G.A., Boutalis, Y.S., Karras, D.A. and Mertzios, B.G. (2007) A New Class of Zernike Moments for Computer Vision Applications. Information Sciences, 177, 2802-2819. https://doi.org/10.1016/j.ins.2007.01.010

[7] Almoosa, N.I., Bae, S.H. and Juang, B.H. (2008) Toward Robust Moment Invariants for Image Registration. Proceedings of IEEE International Conference on Acoustics, Speech and Signal Processing (ICASSP2008), 1009-1012. https://doi.org/10.1109/ICASSP.2008.4517783

[8] Papakostas, G.A., Boutalis, Y.S., Karras, D.A. and Mertzios, B.G. (2007) Fast Numerically Stable Computation of Orthogonal Fourier-Mellin Moments. IET Computer Vision, 1, 11-16. https://doi.org/10.1049/iet-cvi:20060130

[9] Ping, Z.L., Wu, R.G. and Sheng, Y.L. (2002) Describing Image with ChebyshevFourier Moments. J. Opt. Soc. Am., 19, 1748-1754. https://doi.org/10.1364/JOSAA.19.001748

[10] Yap, P.T., Paramesran, R. and Ong, S.H. (2003) Image Analysis by Krawtchouk Moments. IEEE Transactions on Image Processing, 12, 1367-1377. https://doi.org/10.1109/TIP.2003.818019

[11] Amu, G.L. and Hasi, S.R. (2004) Invariant Image Analysis by Pseudo-Jacobi-Fourier Moments. Applied Optics, 43, 2093-2101. https://doi.org/10.1364/AO.43.002093

[12] Bayraktar, B., Berna, T. and Robinson, J.P. (2007) A Numerical Recipe for Accurate Image Reconstruction from Discrete Orthogonal Moments. Pattern Recognition, 40, 659-669. https://doi.org/10.1016/j.patcog.2006.03.009

[13] Broumandnia, A. and Shanbehzadeh, J. (2007) Fast Zernike Wavelet Moments for Farsicharacter Recognition. Image and Vision Computing, 25, 717-726. https://doi.org/10.1016/j.imavis.2006.05.014

[14] Juday, R.D. (1993) Optimal Realizable Filters and the Minimum Euclidean Distance Principle. Applied Optics, 32, 5100-5111. https://doi.org/10.1364/AO.32.005100

[15] Bhatia, A.B. and Wolf, E. (1954) On the Circular Polynomials of Zernike and Related Orthogonal Sets. Pro. Cambridge Philos. Soc., 50, 40-48. https://doi.org/10.1017/S0305004100029066 
Submit or recommend next manuscript to SCIRP and we will provide best service for you:

Accepting pre-submission inquiries through Email, Facebook, LinkedIn, Twitter, etc. A wide selection of journals (inclusive of 9 subjects, more than 200 journals)

Providing 24-hour high-quality service

User-friendly online submission system

Fair and swift peer-review system

Efficient typesetting and proofreading procedure

Display of the result of downloads and visits, as well as the number of cited articles Maximum dissemination of your research work

Submit your manuscript at: http://papersubmission.scirp.org/

Or contactopj@scirp.org 